\title{
Arabidopsis AtDjA3 Null Mutant Shows Increased Sensitivity to Abscisic Acid, Salt, and Osmotic Stress in Germination and Post-germination Stages
}

\author{
Silvia Salas-Muñoz, Aída A. Rodríguez-Hernández, Maria A. Ortega-Amaro, \\ Fatima B. Salazar-Badillo and Juan F. Jiménez-Bremont*
}

Laboratorio de Biotecnología Molecular de Plantas, División de Biología Molecular, Instituto Potosino de Investigación Científica y Tecnológica, San Luis Potosí, México

\section{OPEN ACCESS}

Edited by:

Susana Araújo,

Instituto de Tecnologia Química

e Biológica - Universidade Nova

de Lisboa, Portugal

Reviewed by:

Anca Macovei,

International Rice Research Institute

Philippines

Ji-Hong Liu,

Huazhong Agricultural University,

China

*Correspondence:

Juan F. Jiménez-Bremont

jbremont@ipicyt.edu.mx

Specialty section:

This article was submitted to Crop Science and Horticulture,

a section of the journal

Frontiers in Plant Science

Received: 08 September 2015 Accepted: 09 February 2016

Published: 25 February 2016

Citation:

Salas-Muñoz S,

Rodríguez-Hernández $A A$, Ortega-Amaro MA, Salazar-Badillo FB and Jiménez-Bremont JF (2016) Arabidopsis AtDjA3 Null Mutant

Shows Increased Sensitivity to Abscisic Acid, Salt, and Osmotic Stress in Germination and Post-germination Stages.

Front. Plant Sci. 7:220

doi: 10.3389/fpls.2016.00220

DnaJ proteins are essential co-chaperones involved in abiotic and biotic stress responses. Arabidopsis AtDjA3 gene encodes a molecular co-chaperone of 420 amino acids, which belongs to the J-protein family. In this study, we report the functional characterization of the AtDjA3 gene using the Arabidopsis knockout line designated $j 3$ and the 35S::AtDjA3 overexpression lines. Loss of AtDjA3 function was associated with small seed production. In fact, $j 3$ mutant seeds showed a reduction of $24 \%$ in seed weight compared to Col-0 seeds. Expression analysis showed that the AtDjA3 gene was modulated in response to $\mathrm{NaCl}$, glucose, and abscisic acid (ABA). The $j 3$ line had increased sensitivity to $\mathrm{NaCl}$ and glucose treatments in the germination and cotyledon development in comparison to parental Col-0. Furthermore, the $j 3$ mutant line exhibited higher ABA sensitivity in comparison to parental Col-0 and 35S::AtDjA3 overexpression lines. In addition, we examined the expression of $A B / 3$ gene, which is a central regulator in ABA signaling, in $j 3$ mutant and 35S::AtDjA3 overexpression lines. Under $5 \mu \mathrm{M}$ ABA treatment at $24 \mathrm{~h}, j 3$ mutant seedlings displayed higher $A B / 3$ expression, whereas in 35S::AtDjA3 overexpression lines, AB/3 gene expression was repressed. Taken together, these results demonstrate that the AtDjA3 gene is involved in seed development and abiotic stress tolerance.

Keywords: Arabidopsis thaliana, AtDjA3, abscisic acid, abiotic stress, heat shock proteins, J-protein

\section{INTRODUCTION}

Seed germination and seedling establishment are the most critical stages of survival during the life cycle of an individual plant (Daszkowska-Golec, 2011). Seeds are exposed to a wide range of unfavorable environmental conditions that induce stress, and therefore have a negative impact on germination, growth, and development (Rao et al., 2006). As a result, seeds have developed defense mechanisms that allow them to tolerate and respond rapidly to unfavorable conditions (Koornneef et al., 2002; Vallejo et al., 2010).

Heat Shock Proteins (HSP) are accumulated during abiotic stress as a defense mechanism, and at the later stages of seed development appear to play a protective role in desiccation tolerance 
(Wehmeyer and Vierling, 2000; Koornneef et al., 2002). HSPs are involved in a variety of cellular processes including protein folding, assembly of oligomeric proteins, transport of proteins across membranes, stabilization of polypeptide strands and membranes, and prevention of protein inactivation (Vierling, 1991; Wang et al., 2004; Xue et al., 2010). In plants, HSPs are classified into five classes according to their molecular weight: HSP100, HSP90, HSP70, HSP60, and small HSP (sHSP; Wang et al., 2004).

HSP40 proteins, also referred to as DnaJ or J-proteins, are co-chaperones of the HSP70 machine. J-proteins are key players in stimulating HSP70 ATPase activity, thereby stabilizing its interaction with client proteins (Bukau and Horwich, 1998; Walsh et al., 2004). The J-proteins contain in the N-terminus region a highly conserved domain of approximately 70 amino acids, known as the J domain. This domain consists of four $\alpha$-helices comprising two short helices (I and IV) and two tightly packed anti-parallel helices (II and III) linked by a loop region that contains a highly conserved tri-peptide (HPD: histidine-proline-aspartic acid), which is required for interaction with HSP70 proteins (Wall et al., 1994; Rajan and D'Silva, 2009). Adjacent to J domain is a characteristic glycine and phenylalanine $(\mathrm{G} / \mathrm{F})$ rich region. It has been proposed that this region serves as a flexible linker region and controls the specificity of J-protein functions (Craig et al., 2006). After the G/F-rich region there is a cysteine-rich region which forms a type I zinc-finger domain, which contains four repeated motifs (CXXCXGXG). This domain is essential for binding to unfolded protein and assists HSP70 with protein folding (Bukau and Horwich, 1998; Lu and Cyr, 1998). Finally, the C-terminal region, which is less conserved, is important for providing specificity for HSP70 J-protein machinery (Shi et al., 2005). Classification of J-proteins include the type I or A proteins, which present all the characteristic domains or regions; type II or $\mathrm{B}$ proteins, that lack the zinc-finger domain, and type III or C proteins that only contain domain J (Rajan and D'Silva, 2009). In the Arabidopsis thaliana genome, 116 J-proteins and four J-like proteins have been identified; of which eight belong to type I, 16 to type II, and 92 to type III (Rajan and D'Silva, 2009). The A. thaliana AtDjA3 protein belongs to the type I classification. In plants, J-proteins are induced under different stress conditions. AtDjA3 gene is expressed in roots, stems, leaves, flower buds, flowers, and siliques, and its expression can be induced by heat, cold, and drought stress (Li et al., 2005, 2007), and also under saline conditions with alkaline $\mathrm{pH}$ (Yang et al., 2010).

In the present study, we deepen our understanding of AtDjA3 gene under salt and osmotic stress, and the application of phytohormone abscisic acid (ABA). Expression analysis of the AtDjA3 gene in Arabidopsis seedlings revealed that its expression is modulated by $\mathrm{NaCl}$, glucose, and ABA. For the molecular characterization of AtDjA3 gene, we analyzed the Atdja3-null (j3) mutant and 35S::AtDjA3 overexpression lines. Our results reveal that $j 3$ loss-of-function mutant produces small seeds that are less tolerance to salt and osmotic stress, reflected by a reduced germination rate and lower percentage of green cotyledons in comparison to the Col- 0 and $35 S:: A t D j A 3$ overexpression lines. In addition, the $j 3$ mutant line shows more sensitivity to exogenous ABA. Our results suggest that AtDjA3 gene plays a role in abiotic stress tolerance.

\section{MATERIALS AND METHODS}

\section{Plant Material and Growth Conditions}

The Atdja3 mutant line (j3) and transgenic lines (OvJ3-8 and 14) used in this study were generated in the Arabidopsis thaliana ecotype Columbia 0 (Col-0) background. The T-DNA insertion line (Salk_132923) for the AtDjA3 gene (At3g44110) was obtained from the Salk Institute Genomic Analysis Laboratory ${ }^{1}$ (Alonso et al., 2003). The seeds used for all experiments were harvested at the same time. The seeds of A. thaliana Col-0, Atdja3-null mutant line (j3) and 35S::AtDjA3 overexpression lines (OvJ3) were sterilized with $20 \%(\mathrm{v} / \mathrm{v})$ commercial sodium hypochlorite ( $6 \%$ free chlorine) solution for $5 \mathrm{~min}$, and rinsed five times in sterile distilled water. Aseptic seeds were germinated and grown on agar plates containing Murashige and Skoog (MS) 0.5x medium supplemented with $7 \mathrm{~g} / \mathrm{L}$ phytagel, and $1.5 \%$ sucrose (Murashige and Skoog, 1962). Plates were kept at $4^{\circ} \mathrm{C}$ for 3 days, and then incubated at $22 \pm 2{ }^{\circ} \mathrm{C}$ for 10 days in a growth chamber under a $16 \mathrm{~h}$ light (13,000 luxes)/8 h dark photoperiod. Plants were grown to maturity in soil pots, with a mixture of Sunshine Mix \#3 commercial substrate, perlite and vermiculite (3:1:1), in a growth chamber at $22 \pm 2{ }^{\circ} \mathrm{C}$ with a $16 \mathrm{~h}$ light $(13,000$ luxes $) / 8 \mathrm{~h}$ dark photoperiod.

\section{Nucleic Acids Isolation and cDNA Synthesis}

Genomic DNA was isolated from A. thaliana WT (Col-0) and T-DNA insertion mutant line plants using the method described by Murray and Thompson (1980). RNA extraction from Col-0, Atdja3-null mutant line and 35S::AtDjA3 overexpression lines was performed using the Concert Plant RNA Reagent (Invitrogen, Carlsbad, CA, USA) by following the manufacturer's instructions; samples were stored at $-70^{\circ} \mathrm{C}$ until analysis. For the removal of contaminating genomic DNA, RNA samples were treated with DNase I (Invitrogen, Carlsbad, CA, USA). Synthesis of cDNA was carried out with the Super Script II Reverse Transcriptase enzyme (Invitrogen, Carlsbad, CA, USA) according to the manufacturer's instructions. The cDNAs were stored at $-20^{\circ} \mathrm{C}$ for subsequent use.

\section{Identification of the T-DNA Insertional Mutant Line}

Seeds of T-DNA insertion mutant line (Salk_132923) were germinated in plates containing MS $0.5 \mathrm{x}$ medium; after 10 days they were transferred to soil pots. For genotype analysis PCR assays were performed on genomic DNA isolated from 3-week-old plants using the T-DNA left border (LB) oligonucleotide and gene-specific oligonucleotides designed flanking the T-DNA. The oligonucleotides used were:

\footnotetext{
${ }^{1}$ http://signal.salk.edu
} 
FwSalk_132923 5'-CTTGAAGGTATCTCTTGAGGATGTGT ACC-3' ${ }^{\prime}$, RvSalk_132923 5'-GACGATGCATCTGAATACGTACC AGG-3', and FwLB 5' - AGCAAGCGGTCCACGCTGGTTT-3' .

\section{Generation of $A$. thaliana 35S::AtDjA3 Overexpression Lines}

The AtDjA3 open reading frame (At3g44110 GenBank ID: 823531) was amplified from a cDNA sample of Arabidopsis seedlings using the Hot Start High-Fidelity Polymerase Kit (Qiagen, USA), and the following oligonucleotides: FwAtDjA3 5'-GGCGAAAAGATGTTCGGTAGAGG-3' and RvAtDjA3 5'-GTCTCTCTAAGGAGTTACTTACTGC-3'. The amplified product of $1,263 \mathrm{bp}$ was cloned into the pCR8/GW/TOPO vector (Invitrogen, Carlsbad, CA, USA). The cloned products were sequenced using the M13 oligonucleotide in an ABIPRISM 377 DNA automated sequencer (Perkin Elmer, USA). The sequenced entry clone was recombined into the pMDC32 destination vector (Curtis and Grossniklaus, 2003), by site-specific recombination using the Gateway LR Clonase II Enzyme Mix (Invitrogen, Carlsbad, CA, USA). The pMDC32-AtDjA3 vector was transferred into the Agrobacterium tumefaciens strain GV2260 by electroporation, and transformed into Arabidopsis plants WT (Col-0) by the "floral dip" method (Zhang et al., 2006). Transgenic lines carrying the AtDjA3 gene (35S::AtDjA3) were selected on MS $0.5 \mathrm{x}$ medium containing $50 \mathrm{mg} / \mathrm{mL}$ hygromycin. To verify the expression levels of the AtDjA3 gene in the two transgenic lines, RT-PCR analysis was carried out. For that, we used the following oligonucleotides: FwAtDjA3 $5^{\prime}$-TGACGATGAAGATGATGACCATC- $3^{\prime}$ and RvT-NOS $5^{\prime}$ ATTGCCAAATGTTTGAACGATCG- ${ }^{\prime}$. As loading control, the A. thaliana Actin8 (Atlg49240) transcript was amplified using the FwAct8 5'- GCCAGTGGTCGTACAACCG-3' and RvAct8 5'-CACGACCAGCAAGGTCGAGACG-3' oligonucleotides. The T2 generation of transgenic plants was transferred into soil pots and grown in growth chambers under controlled conditions to produce seeds. Homozygous transgenic lines (T3) were used for the subsequent analysis of seed germination and in the stress tolerance assays.

\section{Quantitative RT-PCR (qRT-PCR) of AtDjA3 Gene Under Abiotic Stress}

Total RNA from $A$. thaliana was obtained from seedlings as described above and used for qRT-PCR assays. Possible genomic DNA contamination was removed using DNase I (Invitrogen, Carlsbad, CA, USA). RNA concentration was measured in a NanoDrop ND-1000 UV-Vis spectrophotometer (NanoDrop Technologies) before and after treatment with DNase I. cDNA synthesis and qRT-PCR analysis were performed by the one-step assay using the Power SYBR ${ }^{\circledR}$ Green RNA-to-CT ${ }^{\mathrm{TM}}$ One-Step Kit (Applied Biosystems, USA). The expression levels of the AtDjA3 gene under salt, osmotic, and exogenous ABA treatments were assessed in 15-day-old A. thaliana Col-0 plants. Seedlings were transferred to liquid MS $0.5 \mathrm{x}$ medium supplemented with $\mathrm{NaCl}$ (150 and $175 \mathrm{mM}$ ), glucose (5 and 6\%), and $\mathrm{ABA}(1,3$, and $5 \mu \mathrm{M})$ for 12 and $24 \mathrm{~h}$. Expression level analyses of the AtDjA3 gene were performed using the following oligonucleotides: FwqRTJ3n 5'-TGACGATGAAGATGATGACCATC- $3^{\prime}$ and RvqRTJ3n $5^{\prime}$-GCAAGAGACAAATTGGTTGGAG- $3^{\prime}$ for the AtDjA3 gene (At3g44110), and UBQ5-F 5'-TCGACGCTTCATCTCGTCCT-3' and UBQ5-R 5'-CGCTGAACCTTTCCAGATCC-3' for the UBQ5 (At3g62250) control gene. The expression level of the $A B I 3$ gene was measured using RNAs obtained from 15-day-old Col-0, Atdja3-null mutant and 35S::AtDjA3-8 overexpression lines that were previously treated with $\mathrm{ABA}(0$ and $5 \mu \mathrm{M}$ ) for 12 and $24 \mathrm{~h}$. Expression level analyses of the $A B I 3$ gene were performed using the following oligonucleotides: FwqABI3 5'-CACAGCCAGAGTTCCTTCCTT- 3' $^{\prime}$ and RvqABI3 $5^{\prime}$-ATGTGGCATGGGACCAGACT-3' for the ABI3 gene (At3g24650), and FwAPT1 5'-GTCATCCCCGACTTCCC

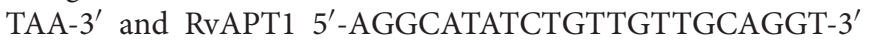
for the APT1 (At1g27450) control gene. cDNA synthesis and quantitative PCR analyses were done in a $10 \mu \mathrm{L}$ reaction mixture containing $50 \mathrm{ng}$ of total RNA as template using the Power SYBR ${ }^{\otimes}$ Green RNA-to-CT ${ }^{\mathrm{TM}}$ 1-Step Kit (Applied Biosystems) as described previously (Salas-Muñoz et al., 2012; Rodríguez-Hernández et al., 2014). For each sample, three biological replicates were analyzed with their respective technical replicates. Experiments were repeated at least twice and gave similar results.

\section{Germination Assays Under Abiotic Stress and Hormone Treatments}

Seeds of A. thaliana ecotype Col-0, Atdja3-null mutant line (j3) and 35S::AtDjA3 overexpression lines (OvJ3; T3) were germinated under different stress conditions. The effect of salt stress on germination was evaluated on MS $0.5 \mathrm{x}$ medium supplemented with 0,125 , and $150 \mathrm{mM} \mathrm{NaCl}$. The effect of osmotic stress on germination was assessed on MS $0.5 x$ medium without sucrose and supplemented with 0,4 , and $5 \%$ glucose. In addition, seeds were germinated in presence of different concentrations of $\mathrm{ABA}(0,1,3$, and $5 \mu \mathrm{M})$. The $\mathrm{ABA}$ stock solution was prepared by dissolving $\mathrm{ABA}$ in small aliquots of $1 \mathrm{~N} \mathrm{NaOH}$. The ABA stock was diluted with distilled water. The germination assays were carried out using 20 seeds per treatment. The seeds were germinated and grown vertically on petri dishes, and counted when the radicle emerged from the seed coat. In addition, the green cotyledon number was scored after 21 days of $\mathrm{NaCl}$, glucose, or ABA treatments. Data are mean $\pm \operatorname{SE}(n=20)$ from five biological replicates. Experiments were repeated at least three times and gave similar results.

\section{Seed Weight Estimation of Col-0, Atdja3-mutant, and 35S::AtDjA3 Over-Expression Lines}

Seed weight was calculated from three replicates for each line $(n=3)$, where 500 seeds represent each replicate. Each seed lot (1-month post-harvest) was measured on an analytical scale, and weights are expressed in milligrams. Experiments were repeated at least three times and gave similar results. 

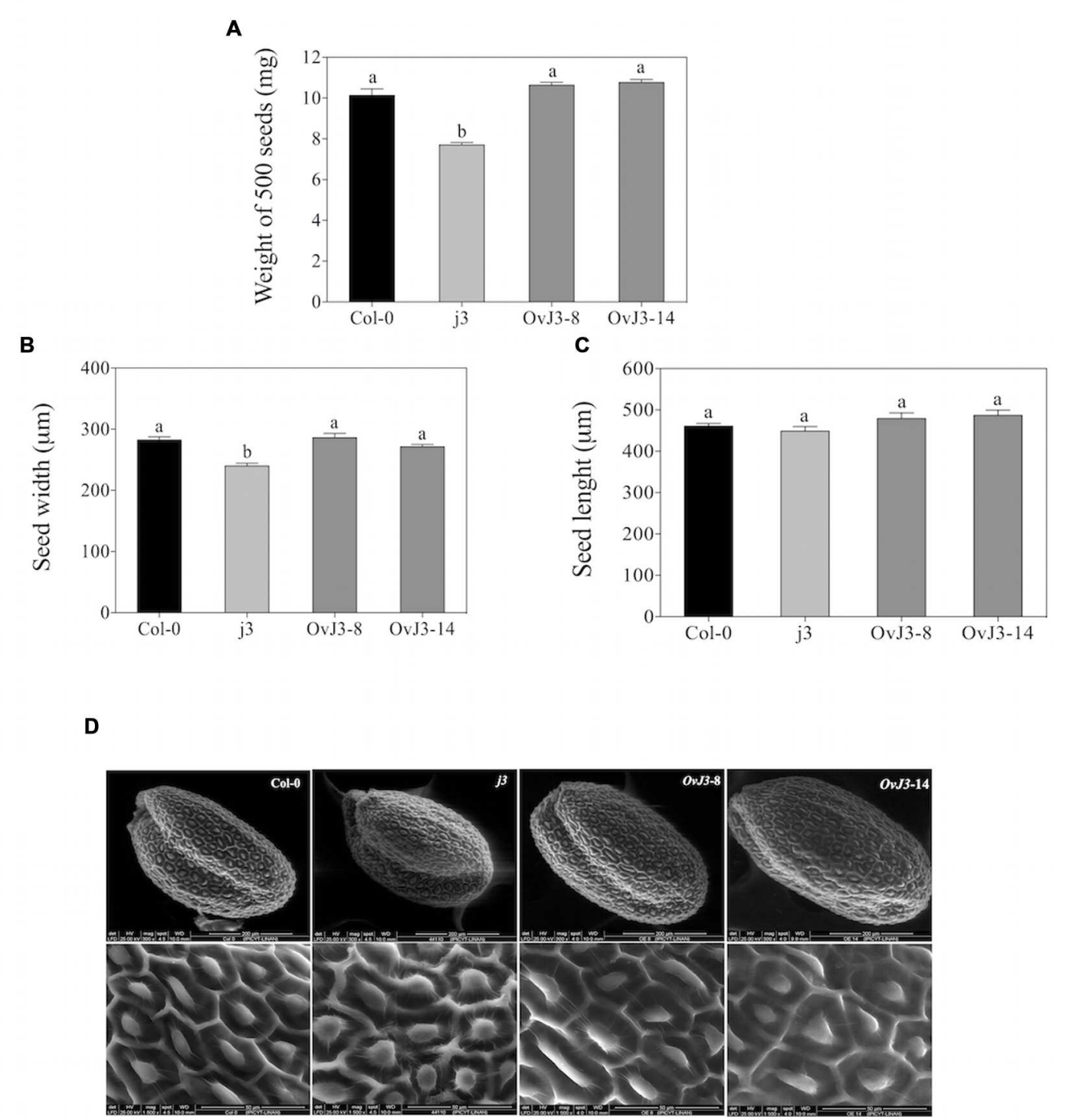

FIGURE 1 | Seed morphology of Atdja3-null mutant line (j3) and 35S::AtDjA3 overexpression lines (OvJ3-8 and -14). (A) Weight of seeds (mg) from the Col-0, j3 mutant line, and 35S::AtDjA3 overexpression lines. Data show means \pm SE from the three groups of 500 dry seeds of each genotype. (B) Width and (C) Length of seeds $(\mu \mathrm{m})$ from the Col-0, 33 mutant line, and 35S::AtDjA3 overexpression lines. (D) Scanning electron micrographs showing: whole seed, scale bar $=200 \mu \mathrm{m}$; seed coat, scale bar $=50 \mu \mathrm{m}$; of Col-0, 33 mutant line, and 35S::AtDjA3 overexpression lines. Error bars represent the means \pm SE $(n=10)$. Different letters indicate significant differences between the Col-0, Atdja3-null mutant line, and 35S::AtDjA3 overexpression lines. One-way ANOVA analyzed significant differences among seeds lines through a Tukey's test $(p \leq 0.05)$.

\section{Microscopic Analysis by Environmental Scanning Electron Microscopy}

For environmental scanning electron microscopy (eSEM) analysis, dried seeds were glued onto pure carbon-containing polymer films, and then fixed onto eSEM sample holders. The external seed morphology of Col-0, Atdja3-null mutant line (j3), and 35S::AtDjA3 overexpression lines $(\mathrm{OvJ} 3)$ were evaluated. The seed width and length were measured with a high-resolution scanning electron microscope (eSEM/QUANTA 200 FEI, Low Vacuum/Water). Morphological seed assays, including width and length of seeds, were carried out using 10 seeds of each genotype.
Photomicrographs were obtained with the eSEM in a pressure chamber at $90-100 \mathrm{~Pa}$ and voltages of 15.0 and $30.0 \mathrm{kV}$.

\section{Statistical Analysis}

To explore potential differences in germination, and green cotyledons among treatments for WT (Col-0), mutant line (j3) and transgenic lines (35S::AtDjA3-8 and -14), we used One-way ANOVA analysis through Tukey's multiple comparison posttest using GraphPad Software. The data are presented as the mean \pm standard error. Differences at $p \leq 0.05$ were considered significant. 


\section{RESULTS}

\section{Seed Morphology of Atdja3-Null Mutant Line (j3) and 35S::AtDjA3 Overexpression Lines (OvJ3)}

To address the biological functions of $A t D j A 3$ gene in seed morphology and response to abiotic stress, mutant, and overexpression lines were characterized. We selected the Salk_132923 line from the Salk T-DNA collection (Alonso et al., 2003), which contains a T-DNA insertion in the fourth exon of AtDjA3 gene (Supplementary Figure S1A). The T-DNA homozygous line was identified by PCR, and the absence of the AtDjA3 transcript was verified by RT-PCR (Supplementary Figure S1B), confirming that the Salk_132923 line is a null allele $(j 3)$ of the $A t D j A 3$ gene. In addition, we generated several transgenic Arabidopsis plants that overexpress the AtDjA3 gene under the control of the CaMV 2X35S promoter (Supplementary Figure S1C). The expression levels of two independent AtDjA3 overexpression lines (35S::AtDjA3-8 and -14) were determined by RT-PCR, observing expression of AtDjA3 gene in all lines analyzed (OvJ3-8 and 14, respectively; Supplementary Figure S1D). Several parameters related to the seed morphology such as weight, length, width, and testa structure were analyzed. We observed that $j 3$ seeds showed a reduction in average seed weight $(7.70 \mathrm{mg} / 500$ seeds) in comparison to Col-0 (10.13 mg/500 seeds). With respect to 35S::AtDjA3-8 and -14 overexpression lines ( $O v J 3-8$ and -14$)$, no significant differences in seed weight between overexpression lines and Col-0 were found (Figure 1A). In order to evaluate the seed width and length, and testa morphology of the Atdja3-null mutant line and 35S::AtDjA3 overexpression lines, micrographs of seeds were taken by eSEM (Figures 1B-D, respectively). In agreement with weight data, the Atdja3-null mutant seeds are reduced in width in comparison to Col-0 and 35S::AtDjA3 overexpression lines (Figure 1B). In addition, the surface of the seed testa in the $j 3$ mutant line displayed variations in the columella shape compared to Col-0 seeds (Figure 1D). With respect to the 35S::AtDjA3 transgenic lines, no significant differences between the overexpression lines and Col-0 were found in seed width and length (Figures 1B,C).

\section{AtDjA3 Gene is Modulated Under Abiotic Stress}

The expression patterns of the AtDjA3 gene in response to salt and osmotic treatments, as well as the application to ABA hormone were assessed. qRT-PCR experiments were carried out in 15-day-old A. thaliana Col-0 seedlings subjected to $\mathrm{NaCl}(0$, 150 , and $175 \mathrm{mM})$, glucose $(0,5$, and $6 \%)$, and $\mathrm{ABA}(0,1,3$, and $5 \mu \mathrm{M}$ ) treatments for 12 and $24 \mathrm{~h}$ (Figure 2). In both salt treatments, an induction of the AtDjA3 gene was observed at 12 and $24 \mathrm{~h}$, except for the $24 \mathrm{~h} 150 \mathrm{mM} \mathrm{NaCl}$ treatment (Figure 2A). With respect to osmotic stress induction by glucose treatments, a slight expression of $A t D j A 3$ gene was observed at both treatment times, achieving the highest expression level with $6 \%$ glucose at $24 \mathrm{~h}$ (Figure 2B). Under ABA treatments, increases in $A t D j A 3$ gene expression were detected with $5 \mu \mathrm{M}$ of hormone
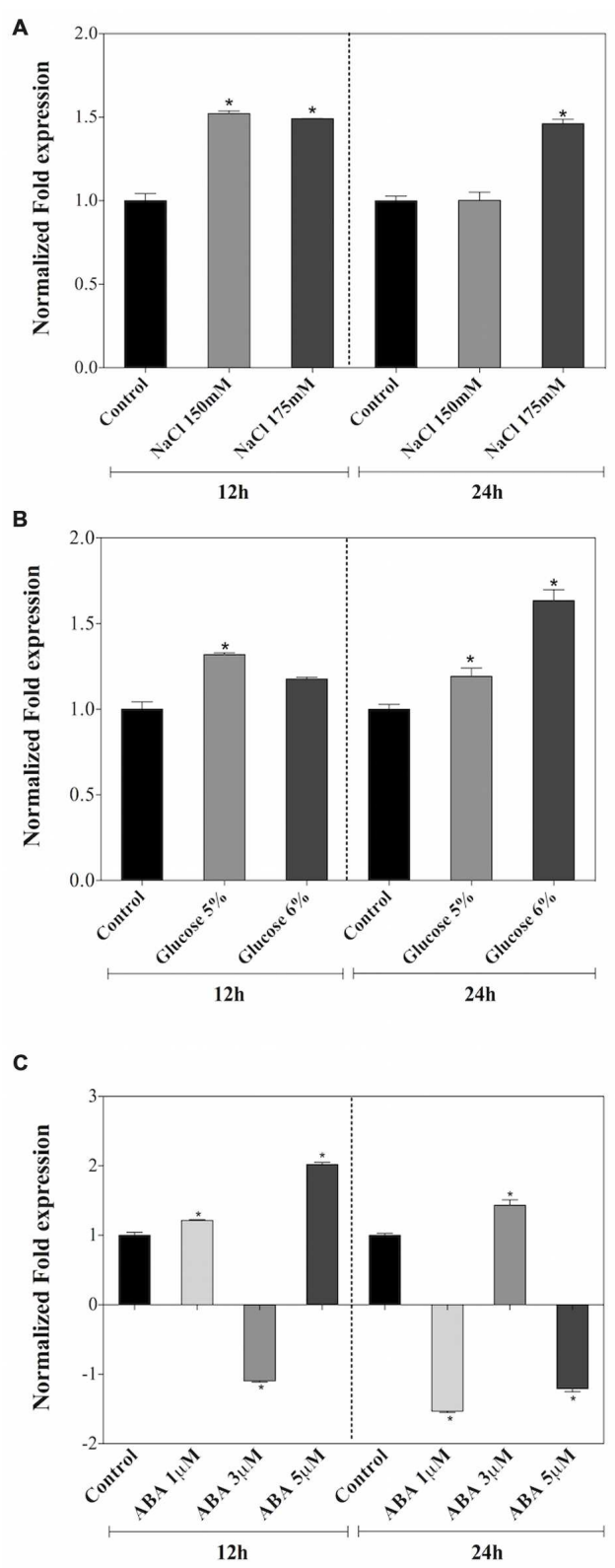

FIGURE 2 | Expression levels of AtDjA3 gene under abiotic stress. The transcript level of AtDjA3 in A. thaliana (Col-0) was determinate in 15-day-old seedlings grown on MS 0.5x liquid medium supplemented with 0, 150, and $175 \mathrm{mM} \mathrm{NaCl}$ (A); 0, 5, and 6\% glucose (B); 0, 1, 3, and $5 \mu \mathrm{M} \mathrm{ABA} \mathrm{(C).}$ Gene expression was determined by qRT-PCR using SYBR green dye. Values represent fold change in expression level upon stressed seedlings compared to non-stressed control seedlings. Quantification was based on a cycle threshold value, with the expression level of the AtDjA3 normalized to the Arabidopsis UBQ5 gene. Bars represent mean \pm SE $(n=3)$. In case of ratios lower than 1, the inverse of the ratio was estimated and the sign was changed. Asterisks indicate statistically significant differences between the samples treated and untreated, according to the One-way ANOVA analysis and multiple comparison Tukey's test $(p \leq 0.05)$.

at $12 \mathrm{~h}$, and $3 \mu \mathrm{M}$ at $24 \mathrm{~h}$ (Figure $2 \mathrm{C}$ ). The results showed that AtDjA3 expression is modulated by abiotic stress. 


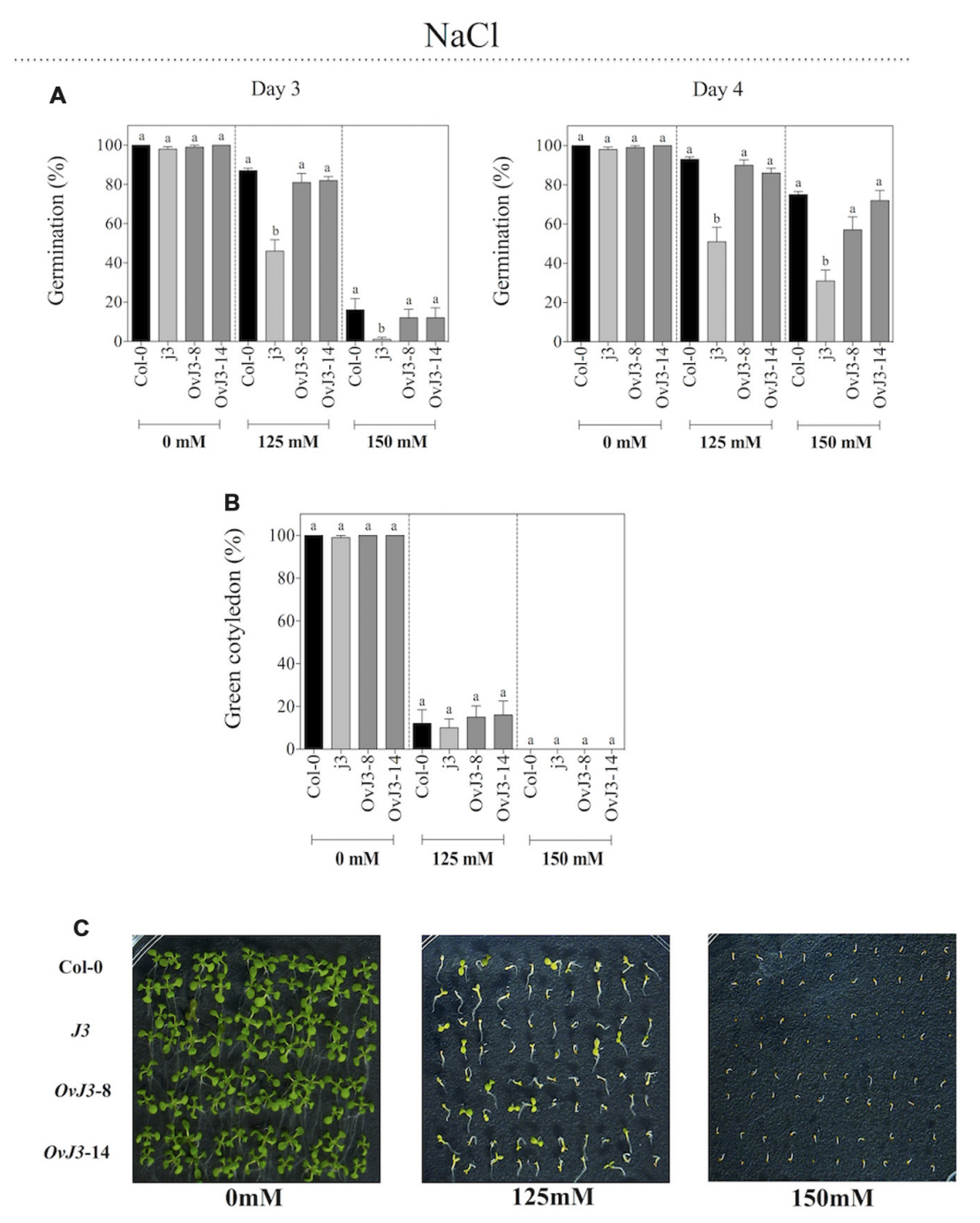

FIGURE 3 | Germination and green cotyledons development of Arabidopsis Col-0, Atdja3-null mutant line (j3), and 35S::AtDjA3 overexpression lines (OvJ3-8 and -14) under salinity. Seeds were germinated on MS 0.5x (1.5\% sucrose) with 0, 125, and 150 mM NaCl. (A) Seed germination was evaluated at 3 and 4 days of treatment. (B) Percent of green cotyledons were recorded, and the phenotype at 21 days after the stress treatment is presented (C). Bars represent the means \pm SE $(n=20)$ of five replicates. Different letters indicate statistically significant differences between the Col-0, Atdja3-null mutant line, and 35S::AtDjA3 overexpression lines. One-way ANOVA was used to analyze the differences among treatments were explored through Tukey's test ( $p \leq 0.05$ ).

\section{The j3 Mutant Line Showed Less Tolerance to Salt Stress in Germination and Post-germination Stages}

To determine whether AtDjA3 plays a role in Arabidopsis tolerance to salt stress, seeds of Col-0, Atdja3-null mutant line (j3) and 35S::AtDjA3 overexpression lines (OvJ3-8 and -14) were germinated on MS $0.5 x$ medium containing 0,125 , and $150 \mathrm{mM} \mathrm{NaCl}$. We observed that $j 3$ mutant line was affected during germination under salt treatments in comparison to Col-0 and the 35S::AtDjA3 overexpression lines (Figure 3A). At 4 days of 125 and $150 \mathrm{mM} \mathrm{NaCl}$ treatments there was a significant decrease in the germination rate of $j 3$ mutant line (51 and 31\%, respectively) compared with Col-0 (93 and $75 \%$, respectively). At 21 days after the germination under salt treatments $(0,125$, and $150 \mathrm{mM})$, the percentage of green cotyledons was recorded (Figures 3B,C). At $125 \mathrm{mM}$ $\mathrm{NaCl}$, no significant differences in green cotyledons among the Col-0, j3 mutant line, and 35S::AtDjA3 overexpression lines were observed (Figures 3B,C). Under control conditions, no significant differences in germination rates and green cotyledons among the Col- $0, j 3$ mutant line, and 35S::AtDjA3 overexpression lines were observed (Figure 3).

\section{The j3 Mutant Line Showed Increased Sensitivity to Glucose in Germination and Post-germination Growth}

In addition to salt treatments, we analyzed the germination rates of Col-0, j3 mutant line, and 35S::AtDjA3 overexpression lines 


\section{Glucose}
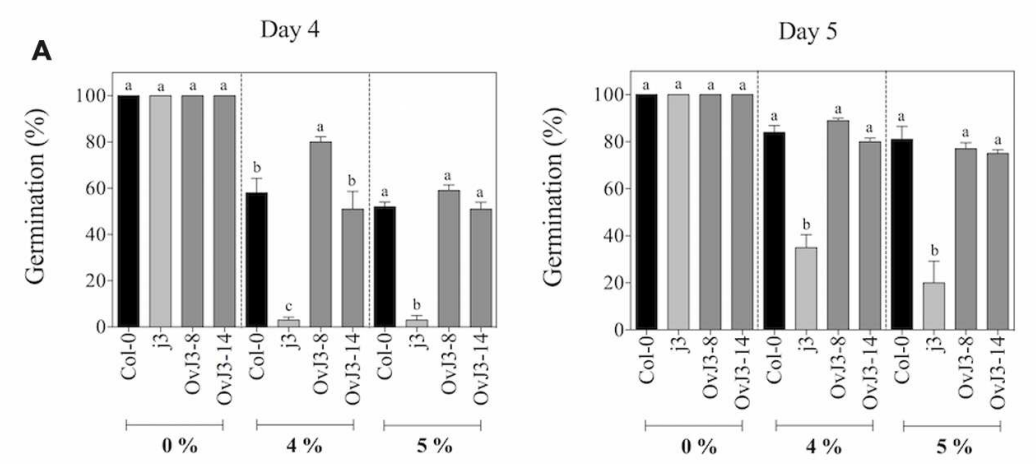

B

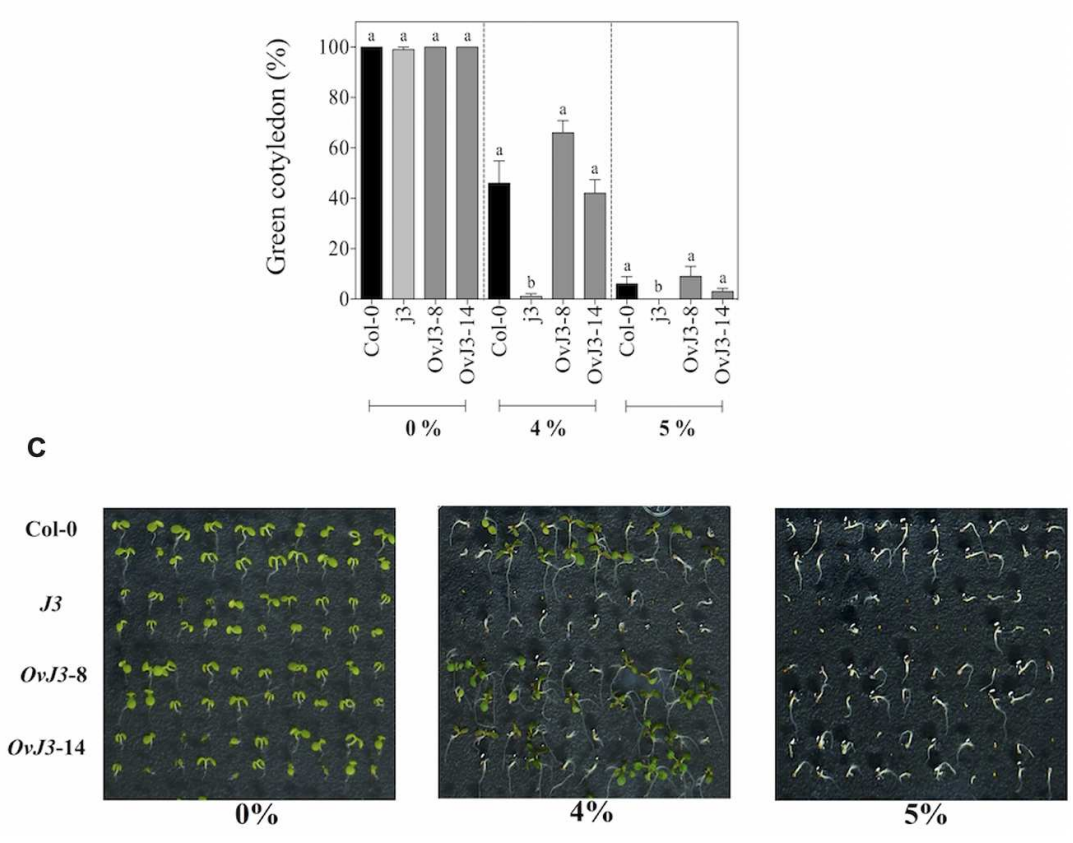

FIGURE 4 | Germination and green cotyledons development of Arabidopsis Col-0, Atdja3-null mutant line (j3), and 35S::AtDjA3 overexpression lines (OvJ3-8 and -14) under osmotic stress. Seeds were germinated on MS 0.5x (without sucrose) 0, 4, and 5\% glucose (A) Seed germination was evaluated at 4 and 5 days of treatment. (B) Percent of green cotyledons were recorded, and the phenotype at 21 days after the stress treatment is presented (C). Bars represent the means \pm SE $(n=20)$ of five replicates. Different letters indicate statistically significant differences between the Col-0, Atdja3-null mutant line, and 35S::AtDjA3 overexpression lines. One-way ANOVA was used to analyze the differences among treatments were explored through Tukey's test $(p \leq 0.05)$.

under osmotic stress, by sowing the seeds on MS $0.5 \mathrm{x}$ medium containing glucose at 0,4 , and $5 \%$ (Figure 4). When seeds were sown on glucose, the $j 3$ mutant line showed a clear reduction in its germination rate with respect to Col-0 and 35S::AtDjA3 overexpression lines (Figure 4). At 4 and 5 days, the osmotic sensitivity was noticed in the $j 3$ mutant line, which achieves only $3 \%$ of germination, whereas the Col-0 and overexpression lines exhibited percentages up to $50 \%$. We analyzed the green cotyledons under glucose treatments (Figures 4B,C). As shown in germination, the $j 3$ mutant line showed an arrest in the development of the green cotyledons at both concentrations (Figure 4C).

\section{The j3 Mutant Line Showed Increased Sensitivity to ABA in Germination and Post-germination Growth}

Inhibitory experiments of seed germination were carried out with Col-0, $j 3$ mutant line, and 35S::AtDjA3 overexpression lines on MS $0.5 x$ medium containing $0,1,3$, and $5 \mu \mathrm{M}$ ABA (Figure 5). The AtDjA3 gene disruption caused a germination sensitivity phenotype on ABA treatments. As observed at 3 and 4 days, the j3 mutant line exhibited the lowest percentage of germination for all of the ABA concentrations assessed (Figure 5A). At the highest concentration of $\mathrm{ABA}(5 \mu \mathrm{M})$, the $j 3$ mutant line did 


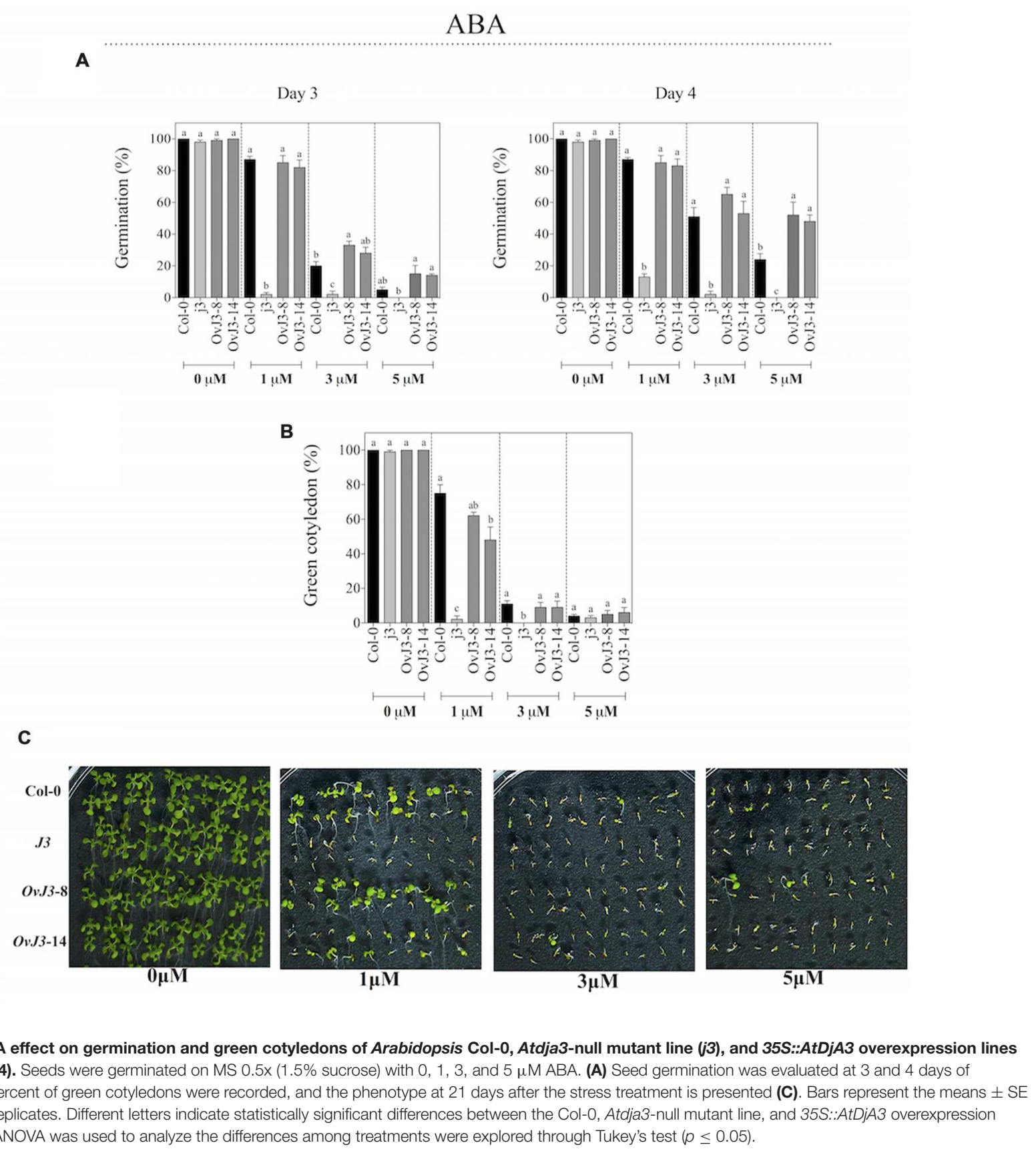

not germinate, whereas the Col- 0 achieved a $24 \%$ germination, and the transgenic lines (35S::AtDjA3) exhibited more than $40 \%$ germination after 4 days (Figure 5A). At 21 days after the germination under $\mathrm{ABA}$ treatment $(0,1,3$, and $5 \mu \mathrm{M})$, the percentage of green cotyledons was recorded (Figures 5B,C). The $j 3$ mutant line exhibits a lower percentage of green cotyledons under ABA treatments. At $1 \mu \mathrm{M} \mathrm{ABA}$, the $j 3$ mutant line showed post-germination growth arrest (only $2 \%$ of green cotyledons), whereas the Col- 0 and the overexpression lines achieved more than $40 \%$ of green cotyledons. These data revealed that the $j 3$ mutant line exhibited an ABA-sensitive phenotype.

\section{Abscisic Acid-Insensitive 3 (AB/3) Gene Expression in the Atdja3-Null Mutant Line (j3) and 35S::AtDjA3 Overexpression Lines (OvJ3) Under ABA Treatment}

Based on ABA sensitivity observed in the $j 3$ mutant line, we examined the expression of $A B I 3$ transcription factor. For this, qRT-PCR expression analyses were carried out in 15-day-old plants of Col-0, 33 mutant line, and 35S::AtDjA3-8 overexpression lines by being subjected to ABA treatment $(5 \mu \mathrm{M})$ for 12 and $24 \mathrm{~h}$ (Figure 6). The $j 3$ mutant line showed higher $A B I 3$ 


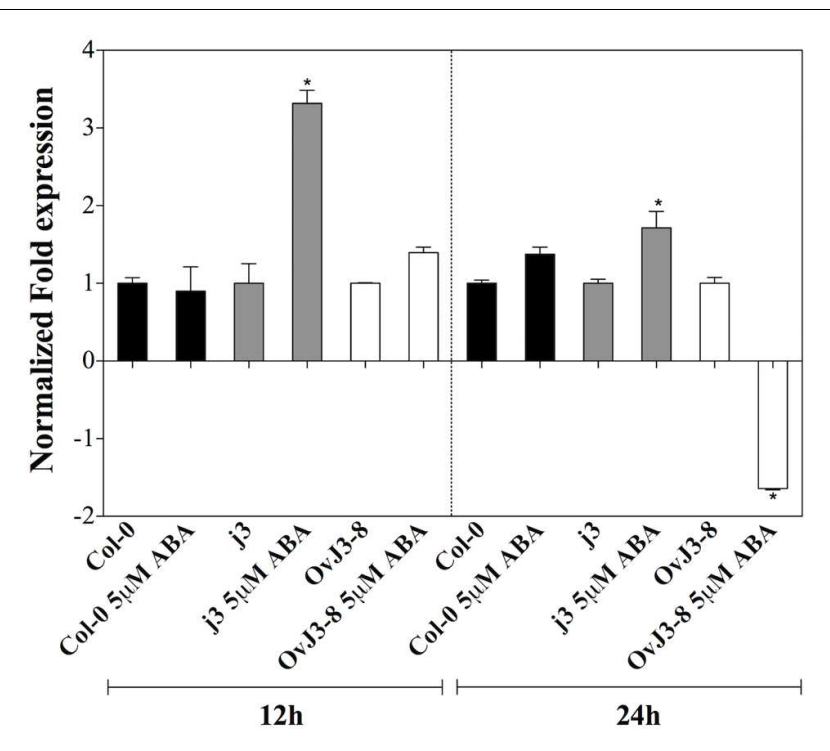

FIGURE 6 | ABI3 expression levels on Arabidopsis Col-0, Atdja3-null mutant line (j3), and 35S::AtDjA3-8 over-expression line under ABA treatment. Fifteen-day-old Arabidopsis seedlings were placed on MS $0.5 \mathrm{x}$ liquid medium supplemented with 0 and $5 \mu \mathrm{M}$ ABA at 12 and $24 \mathrm{~h}$. The expression levels of $A B / 3$ were determined by qRT-PCR using SYBR green dye. Data are expressed as relative mRNA level compared to the plants of each line without ABA treatments, and were calculated after normalization to the Arabidopsis APT1 gene using the comparative threshold method. In case of ratios lower than 1 , the inverse of the ratio was estimated and the sign was changed. Bars represent mean $\pm \mathrm{SE}(n=3)$. Asterisks indicate statistically significant differences between the samples treated and untreated, according to the One-way ANOVA analysis and multiple comparison Tukey's test $(p \leq 0.05)$.

transcript levels under ABA treatment during the entire time course analyzed compared to the parental (Col-0). The highest expression of the $A B I 3$ gene in the mutant line was observed at $12 \mathrm{~h}$ (Figure 6). In contrast, the $A B I 3$ gene was repressed in the 35S::AtDjA3-8 overexpression line at $24 \mathrm{~h}$ under ABA treatment.

\section{DISCUSSION}

One of the major molecular mechanisms to re-establish cellular homeostasis and protect cellular components under abiotic stress is the expression of stress response genes, which encode molecular chaperones such as HSP. HSP40, also known as J-proteins, act as molecular chaperones, and are involved in many cellular processes, including development, signal transduction, and resistance to environmental stresses (Rajan and D'Silva, 2009). We have characterized the Arabidopsis thaliana AtDjA3 gene (At3g44110), which encodes a J-protein, belonging to group I (also known as group A). To determine the possible functional roles of $A t D j A 3$ in response to abiotic stresses, a T-DNA mutant line and overexpression lines (35S::AtDjA3) were obtained and characterized. Remarkably, we found that the Atdja3-null mutant line (j3) produced smaller and lighter seeds in comparison to parental Col-0. On the other hand, seeds from the overexpression lines showed no significant differences in seed weight or size compared to Col-0 seeds. We noticed that the lack of AtDjA3 transcript altered the columella shape of the seeds; these alterations could be due to alterations in the shape and size of seed. In agreement with our proposal, the Arabidopsis microarray database (Arabidopsis eFP Browser ${ }^{2}$ ) reports that AtDjA3 gene is induced during stages 8,9 , and 10 of seed development, and also in dry seed (Schmid et al., 2005). Thus, the loss of function of $A t D j A 3$ gene could be an important factor in seed formation.

We have showed that abiotic stressors modulated the AtDjA3 gene, including $\mathrm{NaCl}$, glucose, and the application of the ABA hormone. The highest accumulation of AtDjA3 transcript was observed under ABA treatment, in particular with $5 \mu \mathrm{M}$ ABA at $12 \mathrm{~h}$. Characterization of the AtDjA3 gene in the germination process of Arabidopsis seeds under abiotic stress conditions using the $j 3$ mutant line and 35S::AtDjA3 overexpression lines revealed that the loss-of-function of $A t D j A 3$ resulted in seeds with sensitivity to salt and osmotic stresses. Furthermore, seeds of the $j 3$ mutant line exhibited increased sensitivity to ABA during germination compared to the parental Col-0. Conversely, 35S::AtDjA3 overexpression lines showed the highest rate of germination after 4 days at the maximum concentration of ABA $(5 \mu \mathrm{M})$. We also examined the effects of the $j 3$ mutant line on the post-germination growth in the salt and osmotic stress response. The results showed that cotyledon development in $j 3$ mutant line was severely inhibited during glucose and ABA treatments, while during salt treatments no differences were observed. On the other hand, the $A t D j A 3$ overexpression lines showed a similar behavior to parental Col-0 in germination and cotyledon greening under salt and osmotic treatments.

A total of $120 \mathrm{~J}$-domain proteins have been identified in the Arabidopsis genome, which represents a large and diverse family of molecular chaperones (Rajan and D'Silva, 2009). Although their functions are mostly uncharacterized, J-proteins have been implicated in plant stress response. In particular, the role of $A t D j A 3$ in heat and salt stress has been documented. For instance, Li et al. (2007) reported that $A t D j A 3$ and its paralogous gene, AtDjA2, improve Arabidopsis thermotolerance. In addition, Yang et al. (2010) showed that plants lacking $A t D j A 3$ gene are more sensitive to salt at alkaline $\mathrm{pH}$, and exhibit decrease plasma membrane $\mathrm{H}^{+}$-ATPase activity. The authors reported that under alkaline conditions, AtDjA3 interacts with protein kinase 5 (PKS5), repressing PKS5 kinase activity to release plasma membrane $\mathrm{H}^{+}$-ATPase. These reports are consistent with our findings, showing that the chaperone AtDjA3 plays a key role during abiotic stress tolerance. Moreover, others J-proteins have been detected during salt and heat stress. For instance, $A N J 1$, a DnaJ gene from Atriplex nummularia, was induced by heat and salt stress (Zhu et al., 1993). Similarly, the expression of SGJ3 (DnaJlike) was rapidly induced in Japanese willow (Salix gilgiana S.) plants upon exposure to heat and salt stress (Futamura et al., 1999). Overexpression of Arabidopsis DnaJ gene (type I,

${ }^{2}$ www.bar.utoronto.ca 
At2g22360) in E. coli and Arabidopsis plants exhibited increased tolerance to $\mathrm{NaCl}$ stress (Zhichang et al., 2010).

We noticed that the disruption of $A t D j A 3$ gene resulted in ABA hypersensitivity. ABA plays an important role in developmental processes such as seed maturation, including synthesis of seed storage proteins and lipids, seed desiccation tolerance, dormancy, control of germination, and the subsequent commitment to seedling growth, and adaptive responses to environmental stimuli in plants (Finkelstein et al., 2002; Cutler et al., 2010; Hubbard et al., 2010; Raghavendra et al., 2010; Fujita et al., 2011). The $j 3$ mutant line showed sensitivity to $\mathrm{ABA}$ in germination and post-germination stages in comparison to parental Col-0. Conversely, 35S::AtDjA3 overexpression lines had less sensitivity to $5 \mu \mathrm{M}$ ABA in germination than those of Col-0. We evaluated the $A B A$-insensitive 3 ( $A B I 3$ ) gene expression in the Col-0, $j 3$ and $35 S:: A t D j A 3$ overexpression lines under $\mathrm{ABA}$ treatment. We found that both the lack of AtDjA3 transcript and its constitutive overexpression altered $A B I 3$ gene expression. $A B I 3$ expression was induced in the $j 3$ mutant line in comparison to WT (Col-0) at 12 and $24 \mathrm{~h}$ after ABA treatment. This increased induction of $A B I 3$ transcript in the $j 3$ mutant line, a key factor in ABA signaling, could be correlated with the ABA hypersensitivity phenotype observed in the $j 3$ mutant line during germination and post-germination growth. The transcriptional factor $\mathrm{ABI} 3$ is considered to be essential for the regulation of seed specific development, so this factor determines the ABA sensitivity and plays a key role in desiccation tolerance and dormancy during zygotic embryogenesis (Zhang et al., 2005). ABI3 transcript and protein levels are abundant in maturing and mature seeds, but disappear soon after germination. However, these levels can be modulated by $\mathrm{ABA}$ or osmotic stress during the time period when postgermination growth arrest occurs (Lopez-Molina et al., 2001, 2002). In contrast with the $j 3$ mutant, we found that transgenic plants overexpressing AtDjA3 were slightly more resistant to $5 \mu \mathrm{M}$ ABA compared to the WT during germination. This could be explained by the down-regulation of the $A B I 3$ transcript at

\section{REFERENCES}

Alonso, J. M., Stepanova, A. N., and Leisse, T. J. (2003). Genome-wide insertional mutagenesis of Arabidopsis thaliana. Science 301, 653-657. doi: 10.1126/science.1086391

Bukau, B., and Horwich, A. L. (1998). The Hsp70 and Hsp60 chaperone machines. Cell 92, 351-366.

Craig, E. A., Huang, P., Aron, R., and Andrew, A. (2006). The diverse roles of J-proteins, the obligate Hsp70 co-chaperone. Rev. Physiol. Biochem. Pharmacol. $156,1-21$.

Curtis, M. D., and Grossniklaus, U. (2003). A gateway cloning vector set for high throughput functional analysis of genes in planta. Plant Physiol. 133, 462-469. doi: $10.1104 /$ pp.103.027979

Cutler, S. R., Rodriguez, P. L., Finkelstein, R. R., and Abrams, S. R. (2010). Abscisic acid: emergence of a core signaling network. Annu. Rev. Plant Biol. 61, 651-679. doi: 10.1146/annurev-arplant-042809-112122

Daszkowska-Golec, A. (2011). Arabidopsis seed germination under abiotic stress as a concert of action of phytohormones. OMICS 15, 763-774. doi: 10.1089/omi.2011.0082

Finkelstein, R. R., Gampala, S. S., and Rock, C. D. (2002). Abscisic acid signalling in seeds and seedlings. Plant Cell 14, S15-S45.
$5 \mu \mathrm{M}$ ABA treatment in 35S::AtDjA3 overexpressing line, which is contrasting behavior to that observed in the Atdja3-null mutant background.

The results presented here showed that the $j 3$ mutant line generates smaller seeds, which were more sensitive to abiotic stress and exogenous application of ABA. This phenotype of $j 3$ mutant line to stress treatments reveals that AtDjA3 gene might have an important role during the germination process, and provides new insights into abiotic stress responses mediated by chaperones.

\section{AUTHOR CONTRIBUTIONS}

SS-M, AR-H, MO-A, FS-B designed and carried out the experiments, analyzed the results, and wrote the manuscript. JJ-B designed the research, contributed scientific advice, correction, wrote and revision of the manuscript. All authors have read and approved the final manuscript.

\section{ACKNOWLEDGMENTS}

This work was supported by the CONACYT: Investigacioin Ciencia Baìsica CB-2013-221075, and Fortalecimiento de infraestructura para la consolidación INFR-2014-01-224800 funds. We are grateful to Dr. Steffen Graether for a grammatical review, and M. C. Alicia Becerra Flora for their technical assistance. We thank M. C. Gladis Delgado Labrada for their technical assistance in the Environmental scanning electron microscopy (eSEM) at LINAN, IPICYT.

\section{SUPPLEMENTARY MATERIAL}

The Supplementary Material for this article can be found online at: http://journal.frontiersin.org/article/10.3389/fpls.2016.00220

Fujita, Y., Fujita, M., Shinozaki, K., and Yamaguchi-Shinozaki, K. (2011). ABAmediated transcriptional regulation in response to osmotic stress in plants. J. Plant Res. 124, 509-525. doi: 10.1007/s10265-011-0412-3

Futamura, N., Ishii-Minami, N., Hayashida, N., and Shinohara, K. (1999). Expression of DnaJ homologs and Hsp70 in the Japanese willow (Salix gilgiana Seemen). Plant Cell Physiol. 40, 524-531. doi: 10.1093/oxfordjournals.pcp.a029573

Hubbard, K. E., Nishimura, N., Hitomi, K., Getzoff, E. D., and Schroeder, J. I. (2010). Early abscisic acid signal transduction mechanisms: newly discovered components and newly emerging questions. Genes Dev. 24, 1695-1708. doi: 10.1101/gad.1953910

Koornneef, M., Bentsink, L., and Hilhorst, H. (2002). Seed dormancy and germination. Curr. Opin. Plant Biol. 5, 33-36. doi: 10.1016/S13695266(01)00219-9

Li, G. L., Chang, H., Li, B., Zhou, W., Sun, D. Y., and Zhou, R. G. (2007). The roles of the atDjA2 and atDjA3 molecular chaperone proteins in improving thermotolerance of Arabidopsis thaliana seedlings. Plant Sci. 173, 408-416. doi: 10.1016/j.plantsci.2007.06.013

Li, G. L., Li, B., Liu, H. T., and Zhou, R. G. (2005). The responses of AtJ2 and AtJ3 gene expression to environmental stresses in Arabidopsis. J. Plant Physiol. Mol. Biol. 31, 47-52. 
Lopez-Molina, L., Mongrand, S., and Chua, N. H. (2001). A post-germination developmental arrest checkpoint is mediated by abscisic acid and requires the ABI5 transcription factor in Arabidopsis. Proc. Natl. Acad. Sci. U.S.A. 98, 4782-4787. doi: 10.1073/pnas.081594298

Lopez-Molina, L., Mongrand, S., McLachlin, D. T., Chait, B. T., and Chua, N. H. (2002). ABI5 acts downstream of ABI3 to execute an ABA- dependent growth arrest during germination. Plant J. 32, 317-328. doi: 10.1046/j.1365313X.2002.01430.x

Lu, Z., and Cyr, D. M. (1998). The conserved carboxyl terminus and zinc finger-like domain of the co-chaperone Ydj1 assist Hsp70 in protein folding. J. Biol. Chem. 273, 5970-5978. doi: 10.1074/jbc.273.10.5970

Murashige, T., and Skoog, F. (1962). A revised medium for rapid growth and bio assays with tobacco tissue cultures. Physiol. Plant. 15, 473-497. doi: 10.1111/j.1399-3054.1962.tb08052.x

Murray, M. G., and Thompson, W. F. (1980). Rapid isolation of high molecular weight plant DNA. Nucleic Acids Res. 8, 4321-4325. doi: 10.1093/nar/8.19.4321

Raghavendra, A. S., Gonugunta, V. K., Christmann, A., and Grill, E. (2010). ABA perception and signaling. Plant Sci. 15, 395-401. doi: 10.1016/j.tplants.2010.04.006

Rajan, V. B. V., and D'Silva, P. (2009). Arabidopsis thaliana J-class heat shock proteins: cellular stress sensors. Funct. Integr. Genomics 9, 433-446. doi: 10.1007/s10142-009-0132-0

Rao, K. M., Raghavendra, A. S., and Reddy, K. J. (eds). 2006). Physiology and Molecular Biology of Stress Tolerance in Plants. Dordrecht: Springer.

Rodríguez-Hernández, A. A., Ortega-Amaro, M. A., Delgado-Sánchez, P., Salinas, J., and Jiménez-Bremont, J. F. (2014). AtGRDP1 gene encoding a glycine-rich domain protein is involved in germination and responds to aba signaling. Plant Mol. Biol. Rep. 32, 1187-1202. doi: 10.1007/s11105-014-0714-4

Salas-Muñoz, S., Gómez-Anduro, G., Delgado-Sánchez, P., Rodríguez-Kessler, M., and Jiménez-Bremont, J. F. (2012). The Opuntia streptacantha OpsHSP18 gene confers salt and osmotic stress tolerance in Arabidopsis thaliana. Int. J. Mol. Sci. 13, 10154-10175. doi: 10.3390/ijms130810154

Schmid, M., Davison, T. S., Henz, S. R., Pape, U. J., Demar, M., Vingron, M., et al. (2005). A gene expression map of Arabidopsis thaliana development. Nat. Genet. 37, 501-506. doi: 10.1038/ng1543

Shi, Y. Y., Hong, X. G., and Wang, C. C. (2005). The C-terminal (331-376) sequence of Escherichia coli DnaJ is essential for dimerization and chaperone activity: a small angle X-ray scattering study in solution. J. Biol. Chem. 280, 22761-22768. doi: 10.1074/jbc.M503643200

Vallejo, A. J., Yanovsky, M. J., and Botto, J. F. (2010). Germination variation in Arabidopsis thaliana accessions under moderate osmotic and salt stresses. Ann. Bot. 106, 833-842. doi: 10.1093/aob/mcq179

Vierling, E. (1991). The roles of heat shock proteins in plants. Annu. Rev. Plant Physiol. Plant Mol. Biol. 42, 579-620. doi: 10.1146/annurev.pp. 42.060191 .003051
Wall, D., Zylicz, M., and Georgopoulos, C. (1994). The NH2-terminal 108 amino acids of the Escherichia coli DnaJ protein stimulate the ATPase activity of DnaK and are sufficient for lambda replication. J. Biol. Chem. 269, 54465451.

Walsh, P., Bursac, D., Law, Y., Cyr, D., and Lithgow, T. (2004). The J-protein family: modulating protein assembly, disassembly and translocation. EMBO Rep. 5, 567-571. doi: 10.1038/sj.embor.7400172

Wang, W., Vinocur, B., Shoseyov, O., and Altman, A. (2004). Role of plant heatshock proteins and molecular chaperones in the abiotic stress response. Trends Plant Sci. 9, 244-252. doi: 10.1016/j.tplants.2004.03.006

Wehmeyer, N., and Vierling, E. (2000). The expression of small heat shock proteins in seeds responds to discrete developmental signals and suggests a general protective role in desiccation tolerance. Plant Physiol. 122, 1099-1108. doi: 10.1104/pp.122.4.1099

Xue, Y., Peng, R., Xiong, A., Li, X., Zha, D., and Yao, Q. (2010). Over-expression of heat shock protein gene hsp26 in Arabidopsis thaliana enhances heat tolerance. Biol. Plant. 54, 105-111. doi: 10.1016/j.jplph.2008.11.013

Yang, Y., Qin, Y., Xie, C., Zhao, F., Zhao, J., Liu, D., et al. (2010). The Arabidopsis chaperone $\mathrm{J} 3$ regulates the plasma membrane $\mathrm{H}+$-ATPase through interaction with the PKS5 Kinase. Plant Cell 22, 1313-1332. doi: 10.1105/tpc.109.0 69609

Zhang, X., Garreton, V., and Chua, N. H. (2005). The AIP2 E3 ligase acts as a novel negative regulator of $\mathrm{ABA}$ signaling by promoting $\mathrm{ABI} 3$ degradation. Genes Dev. 19, 1532-1543. doi: 10.1101/gad.1318705

Zhang, X., Henriques, R., Lin, S. S., Niu, Q. W., and Chua, N. H. (2006). Agrobacterium-mediated transformation of Arabidopsis thaliana using the floral dip method. Nat. Prot. 1, 641-646. doi: 10.1038/nprot.2006.97

Zhichang, Z., Wanrong, Z., Jinping, Y., Jianjun, Z., Xufengand, L. Z. L., and Yang, Y. (2010). Overexpression of Arabidopsis DnaJ (Hsp40) contributes to NaCl-stress tolerance. Afr. J. Biotechnol. 9, 972-978.

Zhu, J. K., Shi, J., Bressan, R. A., and Hasegawa, P. M. (1993). Expression of an Atriplex nummularia gene encoding a protein homologous to the bacterial molecular chaperone DnaJ. Plant Cell 5, 341-349. doi: 10.2307/3869601

Conflict of Interest Statement: The authors declare that the research was conducted in the absence of any commercial or financial relationships that could be construed as a potential conflict of interest.

Copyright (C) 2016 Salas-Muñoz, Rodríguez-Hernández, Ortega-Amaro, SalazarBadillo and Jiménez-Bremont. This is an open-access article distributed under the terms of the Creative Commons Attribution License (CC BY). The use, distribution or reproduction in other forums is permitted, provided the original author (s) or licensor are credited and that the original publication in this journal is cited, in accordance with accepted academic practice. No use, distribution or reproduction is permitted which does not comply with these terms. 